\title{
Enfermedades metabólicas de aparición en la edad adulta Adult-onset metabolic diseases
}

\author{
A. García Ribes ${ }^{1}$, M.J. Martínez González ${ }^{1}$, A. García Cazorla ${ }^{2}$
}

\section{RESUMEN}

Los errores congénitos del metabolismo (ECM) pueden debutar en la adolescencia y en la edad adulta. Aunque es difícil aportar datos exactos de prevalencia ya que existen escasos estudios al respecto, incluso considerándolos poco frecuentes, la importancia de su detección radica en las posibilidades terapéuticas y de consejo genético familiar.

La principal sintomatología de los ECM del adulto es la neurológica, seguida de la hepática. Se puede establecer dos modos básicos de debut. Uno es el agudo, normalmente en forma de alteración del nivel de conciencia, letargia, coma de etiología desconocida en un paciente previamente sano (déficits del ciclo de la urea, trastornos de la remetilación de la homocisteína y porfirias son aquí las causas más frecuentes). Por otra parte está la sintomatología crónica, insidiosa, a menudo progresiva, en la que suele haber cuadros clínicos complejos, y más raramente un síntoma aislado de manera persistente (la enfermedad de Wilson, enfermedades mitocondriales, lisosomales, la enfermedad de Refsum y las glucogenosis son algunos ejemplos en este grupo).

Es de especial importancia conocer las formas de debut agudo, que suelen ser situaciones de extrema urgencia, en las que una conducta adecuada puede evitar el fallecimiento del paciente. En este caso, exámenes sencillos de laboratorio como la determinación del amonio, homocisteína, lactato, acilcarnitinas, aminoácidos, ácidos orgánicos y porfirinas, pueden orientar el diagnóstico y permiten iniciar un tratamiento intensivo.

En este capítulo se pretende realizar un enfoque práctico, abordando las características generales y claves clínicas de sospecha de los ECM más habituales en el adulto.

Palabras clave. Errores congénitos del metabolismo. Edad adulta. Adolescencia

An. Sist. Sanit. Navar. 2008; 31 (Supl. 2): 75-89.

\begin{abstract}
Inborn errors of metabolism (IEM) can have their onset in adolescence or in adulthood. Although it is difficult to contribute exact data on prevalence -because there are few studies in this respect, and IEM are regarded as infrequent- their detection is important due to the possibilities for therapy and family genetic counselling.

The main symptoms of IEM in the adult are neurological, followed by hepatic. Two basic modes of onset can be established. One is acute, normally taking the form of consciousness alteration, lethargy, coma of unknown etiology in a previously healthy patient (urea cycle deficits, homocysteine remethylation disorders and porphyries are the most frequent causes). The other is an insidious, often progressive, chronic symptomathology that can involve complex clinical features, and more rarely a symptom that is isolated in a persistent way (Wilson's disease, mitochondrial diseases, lysosomal storage disorders, Refsum's disease and glycogenosis are some examples of this group).
\end{abstract}

It is especially important to determine the forms of acute onset as these can present situations of extreme emergency where appropriate conduct can prevent the death of the patient. In this case, simple laboratory examinations, such as determination of ammonia, homocysteine, lactate, acylcarnitines, amino acids, organic and porfirines, can guide the diagnosis and enable the start of intensive treatment.

This article provides a practical approach that deals with the general characteristics and the clinical keys for suspecting the most usual IEMs in the adult.

Key words. Inborn errors of metabolism. Adulthood. Adolescence.
1. Unidad de Neuropediatría. Servicio de Pediatría. Hospital de Cruces. Baracaldo. Vizcaya.

2. Servicio de Neurología. Unidad de enfermedades metabólicas. Hospital Sant Joan de Déu. Esplugues. Barcelona.

\section{Correspondencia}

Ainhoa García Ribes

Unidad de Neuropediatría. Servicio de Pediatría Hospital de Cruces

Plaza de Cruces, sn. 48903. Baracaldo. Vizcaya

Tfno. 946006000 Extensión 6484

Fax 946006034

Email: agarcia.ribes@hcru.osakidetza.net 


\section{INTRODUCCIÓN}

Los errores congénitos del metabolismo (ECM) están considerados como enfermedades esencialmente pediátricas. En realidad, el inicio durante las primeras etapas de la vida se describe como rasgo característico de estos trastornos. Probablemente, la aparición precoz viene determinada por el hecho de que las formas pediátricas suelen expresarse de manera más grave y por tanto pueden detectarse con mayor facilidad. Por otra parte, el profesional que atiende a pacientes adultos no suele tener conocimientos ni formación específica en este campo. Por consecuente, lo más habitual es que estos trastornos ni se sospechen ni se diagnostiquen fuera de la edad pediátrica. Es difícil aportar datos de incidencia o prevalencia de estos déficits en el adulto, ya que existen escasos estudios al respecto ${ }^{1}$.

Actualmente, la descripción de nuevas formas de expresión clínica y el mayor conocimiento del que se dispone en este campo, permite detectar con mayor facilidad a aquellos pacientes que inician los síntomas más allá de los 15 años. Este hecho, junto a la mayor supervivencia de los pacientes que inician los síntomas en la infancia, hace que, hoy por hoy, sea imprescindible la existencia de clínicos dedicados a la medicina del adulto, con formación en este área. Neurólogos e internistas serían los principales candidatos para atender a estos pacientes, dada la expresividad clínica de estas enfermedades.

En este capítulo se pretende realizar un enfoque práctico abordando las características generales, principales claves clínicas de sospecha y descripción de los ECM más habituales en la edad adulta.

\section{ECM EN EL NIÑO Y EN EL ADULTO: RASGOS DIFERENCIALES BÁSICOS}

Los trastornos del metabolismo de las moléculas complejas (lisosomales, peroxisomales) y los déficits del metabolismo energético (enfermedades mitocondriales) son los principales grupos de patologías que se presentan en la edad adulta. Cabe destacar además otros trastornos que de manera aislada también se presentan con frecuencia en esta etapa de la vida como la enfermedad de Wilson (metabolismo de los metales) y la enfermedad de Segawa o déficit de GTPCH1 de transmisión dominante (metabolismo de los neurotransmisores).

En el adulto, los ECM suelen aparecer en forma de patologías insidiosas, lentamente progresivas y con mucha menor frecuencia en forma de descompensación aguda o desencadenados por algún agente externo. No obstante, situaciones especialmente catabólicas como el parto, intervenciones quirúrgicas, ejercicio físico intenso o infecciones sistémicas pueden ser los detonantes de estos déficits. Además, el mismo tipo de error congénito puede manifestarse con una clínica totalmente diferente en el adulto que en el niño ${ }^{2}$. La tabla 1 refleja las enfermedades más prevalentes en función del grupo bioquímico al que corresponden, así como las principales diferencias de presentación en función de la edad.

La patología del sistema nervioso suele ser la más representativa en adultos. Las miopatías y trastornos que cursan con hepatoesplenomegalia se hallan también de manera frecuente ${ }^{3}$.

En el adulto es menos probable que en el niño hallar anomalías en las exploraciones complementarias de primera línea (aminoácidos, ácidos orgánicos, lactato, amonio, bioquímica básica) y suelen ser necesarios exámenes más dirigidos y complejos.

\section{MANIFESTACIONES CLÍNICAS DE LOS ECM EN LA EDAD ADULTA}

Estas enfermedades se presentan fundamentalmente como enfermedades neurológicas de diversa expresión aunque en algunos casos de miopatías, manifestaciones oculares $o$, con menor frecuencia, manifestaciones hepáticas aisladas son el primer signo de alarma. La tabla 2 esquematiza algunas de las manifestaciones clínicas, métodos diagnósticos y posiblidades terapéuticas en estos déficits.

Las figuras 1 y 2 ofrecen un algoritmo de aproximación diagnóstica a los ECM del adulto que se ponen de manifiesto con un cuadro de epilepsia o neuropatía periférica 
Tabla 1. Errores congénitos del metabolismo.

\begin{tabular}{|c|c|c|}
\hline Grupos de enfermedades & Manifestaciones en el adulto & Manifestaciones en el niño \\
\hline \multicolumn{3}{|l|}{ Lisosomales } \\
\hline Tay-Sach's, Sandhoff & $\begin{array}{l}\text { Enfermedad de segunda moto- } \\
\text { neurona, distonía, neuronopatía } \\
\text { bulboespinal }\end{array}$ & $\begin{array}{l}\text { Hipotonía, retraso psicomotor, } \\
\text { convulsiones, ceguera }\end{array}$ \\
\hline Leucodistrofia metacromática & $\begin{array}{l}\text { Demencia, trastornos de con- } \\
\text { ducta }\end{array}$ & $\begin{array}{l}\text { Retraso psicomotor, paraparesia } \\
\text { espástica, neuropatía periférica }\end{array}$ \\
\hline $\begin{array}{l}\text { Glicogenosis } \\
\text { Déficit de maltasa ácida II }\end{array}$ & Glicogenosis & Hipotonía, cardiomiopatía \\
\hline $\begin{array}{l}\text { Peroxisomales } \\
\text { Adrenoleucodistrofia-X }\end{array}$ & $\begin{array}{l}\text { Patología desmielinizante medu- } \\
\text { lar y en nervio periférico preser- } \\
\text { vando SNC }\end{array}$ & $\begin{array}{l}\text { Regresión neurológica, parapa- } \\
\text { resia espástica progresiva, leu- } \\
\text { codistrofia, insuficiencia supra- } \\
\text { rrenal }\end{array}$ \\
\hline \multicolumn{3}{|l|}{ Moleculas pequeñas } \\
\hline Trastornos ciclo de la urea & Cuadros psiquiátricos, coma & $\begin{array}{l}\text { Intoxicación neurológica aguda, } \\
\text { síndrome de Reye }\end{array}$ \\
\hline Homocistinuria & Miopía, trombosis & $\begin{array}{l}\text { Retraso cognitivo, luxación cris- } \\
\text { talino }\end{array}$ \\
\hline $\begin{array}{l}\text { Acidos grasos } \\
\text { Déficits beta-oxidación }\end{array}$ & $\begin{array}{l}\text { Mioglobinuria, intolerancia al } \\
\text { ejercicio, muerte súbita por car- } \\
\text { diomiopatía }\end{array}$ & $\begin{array}{l}\text { Hipoglicemias, otras manifesta- } \\
\text { ciones diversas según el tipo de } \\
\text { déficit }\end{array}$ \\
\hline $\begin{array}{l}\text { Neurotranmisores: } \\
\text { Déficit tirosina hidroxilasa }\end{array}$ & Distonía dopa sensible & Encefalopatía grave \\
\hline Mitocondriales & $\begin{array}{l}\text { Mayor incidencia de alteracio- } \\
\text { nes del ADN mitocondrial: } \\
\text { LHON, NARP, MELAS, MERRF y } \\
\text { de miopatías tardías más benig- } \\
\text { nas que en el niño } \\
\text { Lactato, aminoácidos y ácidos } \\
\text { orgánicos son con más frecuen- } \\
\text { cia normales }\end{array}$ & $\begin{array}{l}\text { Afectación multiorgánica, pro- } \\
\text { gresiva, peor pronóstico en } \\
\text { cuanto a esperanza y calidad de } \\
\text { vida. } \\
\text { Con más frecuencia se halla } \\
\text { hiperlactacidemia y alteraciones } \\
\text { de los aminoácidos y ácidos } \\
\text { orgánicos. }\end{array}$ \\
\hline
\end{tabular}

ya que ambas pueden aparecer en el curso del trastorno o ser el síntoma inicial ${ }^{4,5}$.

\section{Trastorno de los aminoácidos y de los ácidos orgánicos}

Los defectos del ciclo de la urea como el déficit de OTC o la citrulinemia se pueden presentar en adultos como episodios intermitentes de coma o alteraciones del comportamiento que deben diferenciarse de trastornos psiquiátricos ${ }^{1,2}$.

Los defectos de la oxidación de los ácidos grasos generalmente se presentan en la infancia con hipoglucemia, pudiendo ser severa. Los adultos también pueden llegar a la hipoglucemia, aunque son más resistentes al ayuno que los niños. Una historia detallada puede revelar la existencia de estos episodios en la infancia. Los defectos de ácidos grasos de cadena larga y el déficit de CPT II (carnitín palmitoil transferasa) pueden presentarse con mioglobinuria y rabdomiolisis después de un ejercicio intenso, debido a la incapacidad del músculo esquelético de utilizar los ácidos grasos para la producción de energía. El defec- 


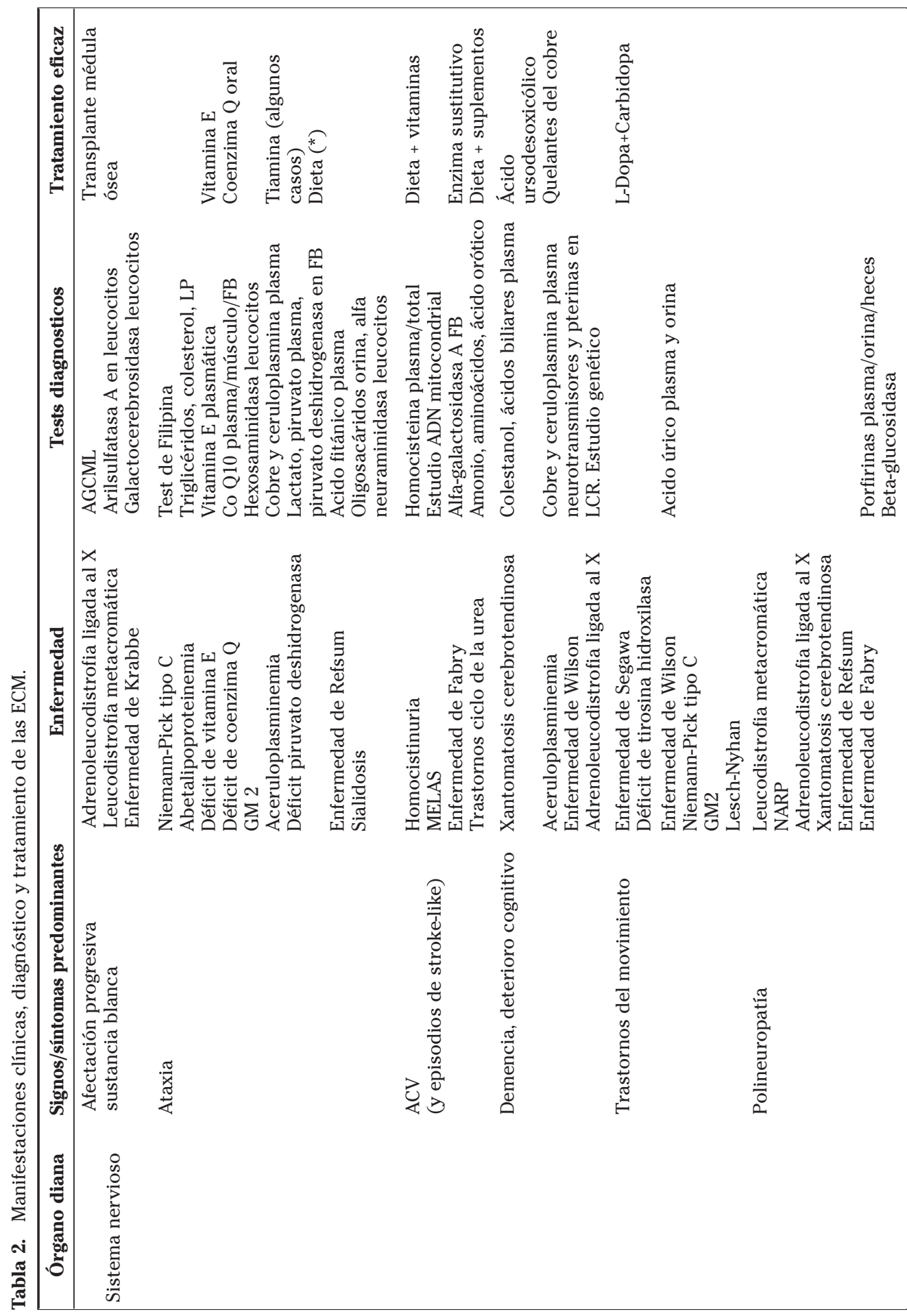




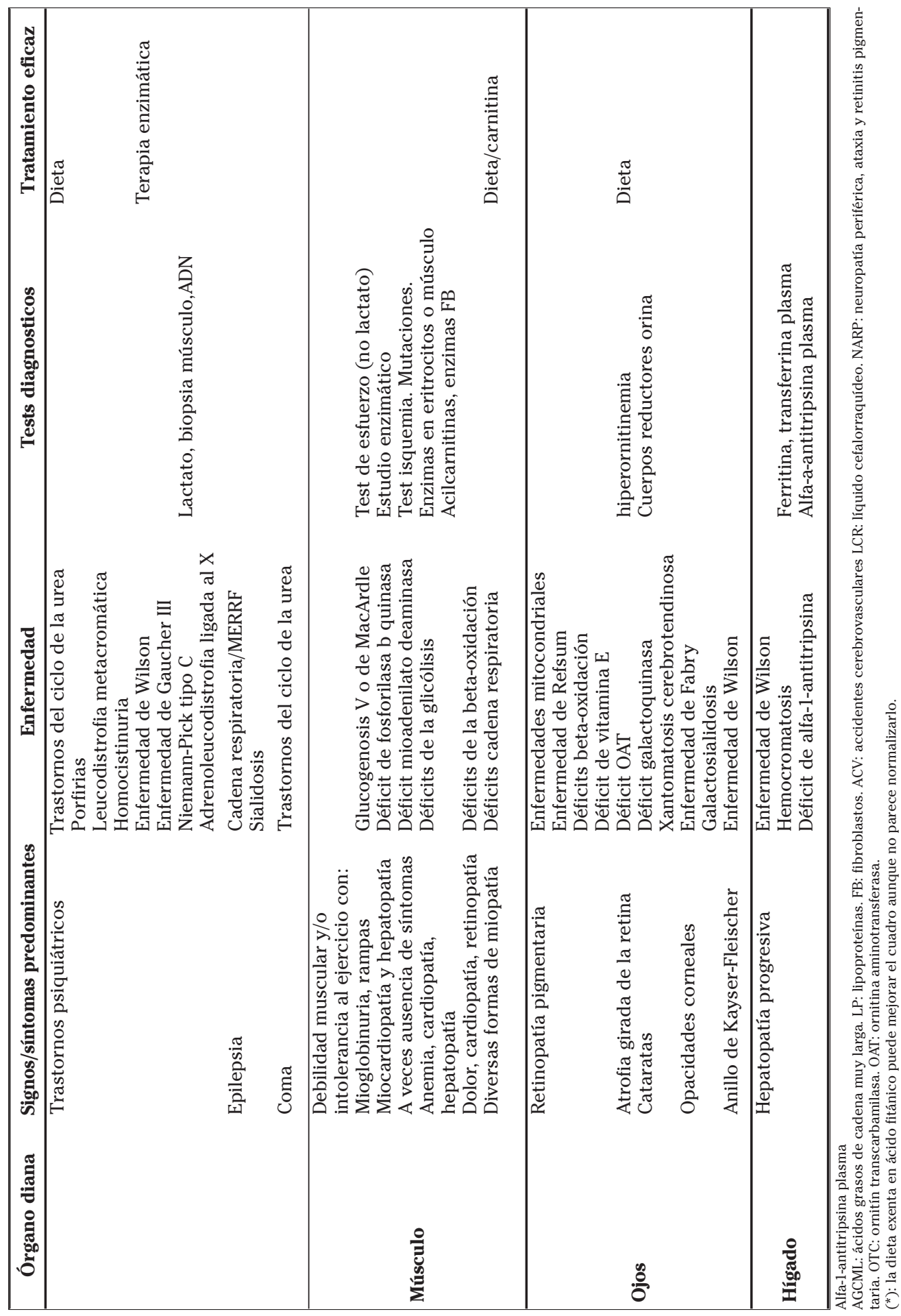


to del trasportador de la carnitina provoca una concentración baja de carnitina en el músculo cardiaco, que causa la muerte súbita por miocardiopatía hipertrófica. La mayoría de estos defectos de la oxidación de los ácidos grasos son tratables, de ahí la importancia del diagnóstico precoz.

La homocistinuria es el error congénito del metabolismo de los aminoácidos sulfurados más frecuente. La forma clásica de la enfermedad se debe al déficit de la cistationina beta sintasa. En adultos puede debutar más allá de los 40 años en forma de miopía, historia de episodios trombóticos, hallazgos marfanoides y retraso men$\mathrm{tal}^{6,7}$. Un signo diagnóstico útil es la presencia de ectopia lentis. Hay una respuesta favorable a la vitamina B6 en algunos casos, por lo que diagnosticar la enfermedad en etapas tempranas es importante.

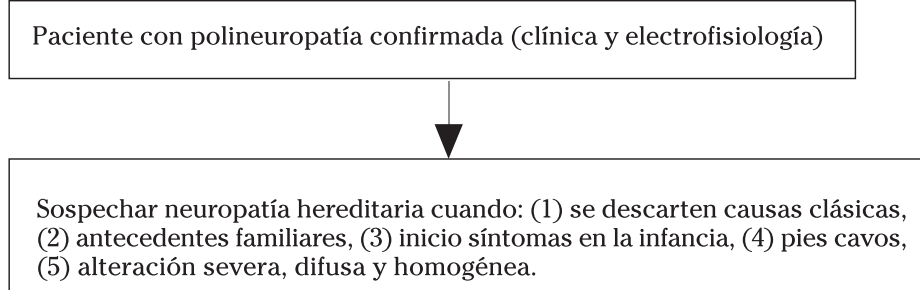

\section{$\checkmark$}

Sospechar ECM cuando: (1) se excluyan los genes habituales, (2) síntomas fluctuantes, (3) herencia recesiva o ligada a X, (4) cuadro clínico difuso con otra sintomatología asociada.

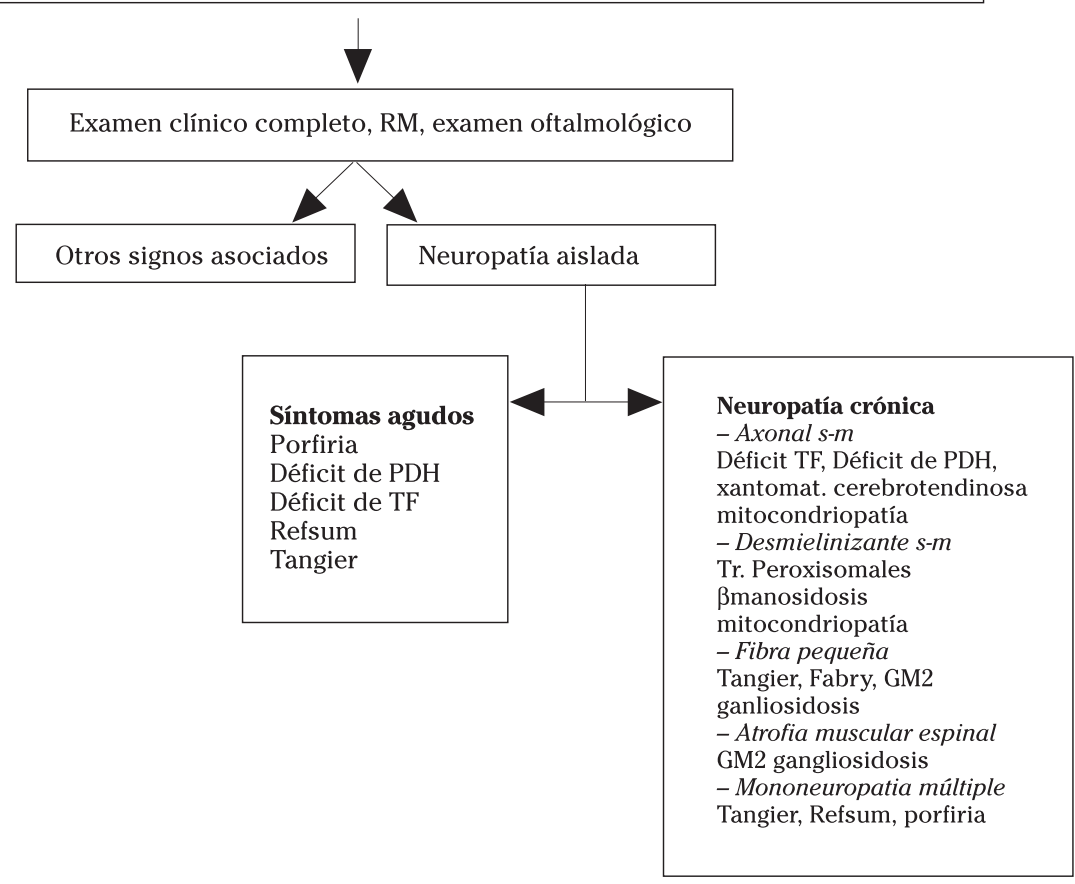

Figura 1. Algoritmo diagnóstico de ECM con neuropatía periférica como forma de presentacion. 


\section{Enfermedades lisosomales}

El lisosoma es una organela intracelular cuya función consiste en la degradación de complejos lipídicos, glicoproteínas, y mucopolisacáridos. Las enfermedades lisosomales son trastornos hereditarios que se producen por la incapacidad de degradar las macromoléculas por un defecto funcional específico. Éste produce la acumulación de metabolitos intermedios. Aunque este proceso ocurre intraútero, la edad de inicio de la clínica puede variar debido a la posibilidad de que haya actividad enzimática funcional residual, que provoque una acumulación más baja de metabolitos intermedios. En las formas de inicio en la edad adulta de estas enfermedades, los hallazgos clínicos pueden diferir sustancialmente de las formas de inicio en la infancia, lo que puede dificultar considerablemente el diagnóstico.

La leucodistrofia metacromática es una enfermedad autosómica recesiva, debida al déficit congénito de la enzima lisosomal arilsultasa A. Este enzima degrada diversos esfingolípidos sulfatados, siendo el cerebrósido sulfato el más importante de ellos, con un papel importante en la composición de la mielina del sistema nervioso central y periférico. Según la edad de presentación existen varios tipos clínicos, siendo la forma más frecuente la infantil tardía, y la forma adulta, la variedad menos descrita en la literatura. Aparecen los primeros síntomas a partir de los 15 años de edad, describiéndose casos cuya clínica se inició a partir de los 40 años e incluso a los $60^{8}$. Se presentan como trastornos de la

Paciente con epilepsia confirmada (clínica y electrofisiología)

Sospechar ECM cuando: (1) el síndrome epiléptico no se corresponde con ningúno conocido (2) epilepsia mioclonica progresiva (3) asociación con otros signos neurológicos, retraso mental inexplicable u alteración en otros órganos (4) historia familiar sugestiva (5) convulsiones relacionadas con las horas de las comidas (6) ineficacia o empeoramiento con el tratamiento anticomicial (7) estatus epiléptico inexplicable (8) alteraciones en la neuroimagen con espectroscopia (9) actividad eléctrica lenta o respuesta a la fotoestimulación a bajas frecuencias

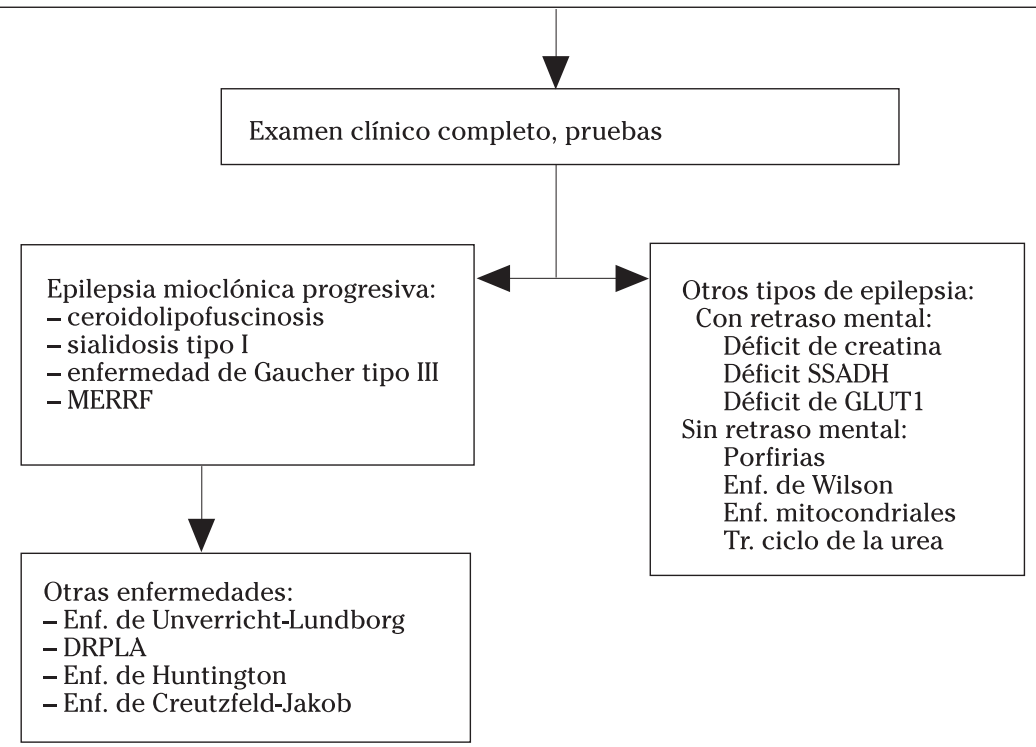

Figura 2. Algoritmo diagnóstico de ECM con epilepsia como forma de presentacion. 
conducta que hay que diferenciar de cuadros de esquizofrenia u otras psicosis, y con un deterioro mental progresivo. Con el avance de la enfermedad aparecen trastornos de la marcha, ataxia y/o neuropatía periférica. Un $25 \%$ de los casos descritos presentaron convulsiones. Este subtipo clínico evoluciona muy lentamente y de manera progresiva, falleciendo entre $10 \mathrm{y}$ 30 años después del inicio de los primeros síntomas. En las pruebas diagnósticas se objetiva una hiperproteinorraquia, siendo ésta de menor intensidad en esta forma que en la infantil tardía. En gran parte de los pacientes la velocidad de conducción neural periférica esta disminuida, aunque en menor magnitud a la detectada en los de la forma infantil tardía, pudiendo preceder a los síntomas secundarios de afectación del sistema nervioso central. En la resonancia magnética (RM) cerebral, se observa una alteración simétrica de la sustancia blanca a diferentes niveles, siendo más marcada en la región cerebral anterior, tanto en esta forma como en la juvenil.

La "pseudodeficiencia de arilsulfatasa A" se da cuando existe un déficit de esta enzima manteniendo una actividad enzimática que oscila entre el 5 y el $50 \%$ de los valores habituales en la población normal. La frecuencia descrita oscila alrededor del $10 \%$. En nuestro país se encuentra en un $12,7 \%$ de la población la presencia de al menos un alelo para la pseudodeficiencia. Debido a su alta frecuencia, podemos encontrar cuadros neurológicos diferentes a la leucodistrofia metacromática en portadores de pseudodeficiencia de arilsulfatasa $\mathrm{A}$, e incluso se pueden asociar a otras leucodistrofias dificultando su diagnóstico. El diagnóstico de leucodistrofia metacromática debe incluir un cuadro clínico compatible, demostración del déficit de arilsulfatasa A, objetivar los depósitos de sulfatide y/o descartar la pseudodeficiencia de arilsulfatasa A mediante estudio molecular.

La enfermedad de Krabbe o leucodistrofia de las células globoideas es una enfermedad autosómica recesiva, secundaria al déficit de la enzima galactosilcerebrosidasa, que provoca un depósito anormal del esfingolípido en el sistema nervioso central y periférico. La forma de inicio en la edad adulta es la menos frecuente, apareciendo los primeros síntomas a partir de los 10 años, habiéndose descrito casos de inicio alrededor de los 40 años ${ }^{9}$. Habitualmente se manifiesta en forma de parestesias y una disminución progresiva de la fuerza en las extremidades inferiores, con progresiva aparición de ataxia cerebelosa, temblor, disminución de la agudeza visual, y deterioro cognitivo. La evolución es lentamente progresiva.

La enfermedad de Tay-Sach's y Sandhoff's son gangliosidosis, que aparecen en la infancia debutando con convulsiones, ceguera, hipotonía y retraso psicomotor. Las formas de inicio en la edad adulta se presentan con un rango clínico muy amplio variando desde formas atípicas de enfermedad de la motoneurona a distonia y neuropatía bulboespinal.

El déficit de maltasa ácida tipo II es una glucogenosis, que se presenta generalmente en las primeras semanas de la vida en forma de hipotonía y miocardiopatía hipertrófica. En el adulto puede iniciarse como una debilidad muscular, pero sin miocardiopatía ${ }^{10}$.

\section{Enfermedades peroxisomales}

Las enfermedades peroxisomales son un grupo creciente de ECM caracterizados por el funcionamiento defectuoso de una o varias enzimas de dicha organela. La importancia de estas enfermedades reside en la variedad de fenotipos clínicos con distinta forma de presentación y en la posibilidad de que debuten desde el periodo neonatal hasta la edad adulta ${ }^{11}$.

Clásicamente los trastornos peroxisomales se dividen en dos grupos, aquellos con deficiencias en la biogénesis, en los que los peroxisomas fracasan en su formación, y los debidos a deficiencias de enzimas únicas que presentan fallo de una sola función, manteniendo la estructura peroxisomal intacta ${ }^{12}$. Los ECM que pueden debutar en la edad adulta se encuentran englobados en el segundo grupo como es el caso de la enfermedad de Refsum del adulto y la adrenoleucodistrofia ligada al X que, por otro lado, es la enfermedad de este grupo más frecuente. Existen formas atenuadas con sintomatología menos severa y evolución más lenta que las formas clásicas 
pediátricas del síndrome de Zellweger o la condrodisplasia rizomiélica punctata, cuyos pacientes pueden alcanzar la edad adulta, no habiéndose descrito formas de presentación en esta edad ${ }^{2}$.

La enfermedad de Refsum del adulto es una enfermedad autosómica recesiva de baja incidencia. Los síntomas aparecen entre el final de la primera década y la edad adulta, desarrollando la triada clásica de retinitis pigmentosa, ataxia cerebelosa y polineuropatía sensitivomotora de predominio distal. Puede haber afectación de los pares craneales, signos cardiacos, dermatológicos y esqueléticos, presentando un curso lentamente progresivo. Los estudios de neuroimagen son normales, siendo característica la presencia de hiperproteinorraquia sin pleocitosis. Para su diagnóstico es preciso que exista una elevación constante del acido fitánico en plasma y tejidos siendo el resto de funciones peroxisomales normales ${ }^{13}$.

La adrenoleucodistrofia ligada al $\mathrm{X}$ (ALD-X) es el trastorno peroxisomal más frecuente. Se debe a un defecto en las proteínas de la membrana peroxisomal que se encargan del primer escalón de la degradación de los ácidos grasos de cadena muy larga (AGCML). Estos ácidos grasos son parte importante de los lípidos del SNC y su acúmulo se asocia a desmielinización de la sustancia blanca cerebral, anomalías del nervio periférico y atrofia de la corteza suparrenal. Aproximadamente dos tercios de los pacientes varones con trastorno neurológico presentan también insuficiencia adrenocortical y/o testicular, que puede preceder, acompañar o seguir al inicio de los síntomas neurológicos.

Podemos diferenciar 6 fenotipos distintos de esta enfermedad, de los cuales el $80 \%$ se corresponden con la forma infantil de adrenoleucodistrofia (ALD) y el resto corresponde la adrenomieloneuropatía (AMN) de inicio en la edad adulta. Existen dos formas clínicas de ALD de inicio en la adolescencia y edad adulta, con características similares a la forma desmielinizante cerebral infantil. Estas formas del adulto tienen una evolución rápidamente progresiva hacia cuadriplejia espástica, estado vegetativo $\mathrm{y}$ muerte en pocos años. La AMN es considerada globalmente la forma fenotípica más frecuente de ALD-X. Los síntomas comienzan en la tercera-cuarta década de la vida con paraparesia progresiva, compromiso sensorial afectando la sensibilidad vibratoria y esfínteres. Aproximadamente en el $50 \%$ de los varones afectos hay afectación del SNC, que puede ponerse de manifiesto años después del inicio de la enfermedad. La esperanza de vida suele ser normal con una evolución lentamente progresiva salvo en casos con desmielinización cerebral en los que el pronóstico empeora, o aquellos con insuficiencia adrenocortical no tratada ${ }^{14}$.

Otra forma de presentación menos frecuente es la variante que cursa con síndrome de Addison aislado, que puede presentarse en la edad adulta y hasta en un 50\% asocia signos neurológicos en algún momento de su evolución. Se piensa que estas formas están infradiagnosticadas, por lo que en aquellos pacientes con disfunción adrenal sin clínica neurológica, pero con un familiar afecto de un trastorno desmielinizante, o en aquellos en los que no se llegue a establecer un diagnóstico claro, se deben estudiar los ácidos grasos de cadena muy larga $a^{2,14,15}$. En la tabla 3 vemos resumidas algunas de las características de estas formas de presentación del adulto.

Los pacientes heterocigotos presentan síntomas neurológicos aproximadamente en el $20-50 \%$ de los casos, los síntomas suelen ser más leves, conservando la función corticoadrenal, y la evolución es más lenta ${ }^{16}$.

Bioquímicamente presentan un acúmulo de los AGCML en plasma, fibroblastos y tejidos afectados, aunque en el 15\% de las mujeres heterocigotas los niveles son normales siendo necesario el estudio molecu$\operatorname{lar}^{17,18}$.

\section{Glucogenosis}

Existen numerosos ECM como consecuencia de mutaciones en genes implicados en prácticamente todas las proteínas que participan en la síntesis, degradación o regulación del glucógeno. Estos defectos genéticos originan un amplio abanico de enfermedades que pueden afectar a células de distintos tejidos, por lo que presentan 
Tabla 3. Enfermedades peroxisomales con formas de presentación en el adulto.

\begin{tabular}{|c|c|c|c|c|}
\hline & ALD Aldol & ALD Adul & AMN & Addison \\
\hline Edad inicio & 10-21 años & $>21$ años & $>18\left(3^{\mathrm{a}}-4\right.$ década $)$ & $>2$ años \\
\hline $\begin{array}{c}\text { Trastorno del } \\
\text { comportamiento }\end{array}$ & + & + & - & - \\
\hline Trastorno cognitivo & + & Frecuente & - & - \\
\hline S. piramidal & Ocasional & Frecuente & Constante & - \\
\hline Afectación RNM SNC & $\begin{array}{c}\text { Extenso en } \\
\text { sustancia blanca } \\
\text { occipital }\end{array}$ & $\begin{array}{c}\text { Extenso en } \\
\text { sustancia blanca } \\
\text { occipital }\end{array}$ & $\begin{array}{l}\text { Cápsula interna, } \\
\text { ganglios base, } \\
\text { mesencéfalo }\end{array}$ & Ausente o leve \\
\hline Polineuropatía & Raro & Posible & Sensitivomotora & - \\
\hline $\begin{array}{c}\text { Potenciales evocados } \\
\text { alterados }\end{array}$ & PEV, PEAT & $\begin{array}{c}\text { PEV, PEAT. } \\
\text { Ocasionalmente } \\
\text { PEES }\end{array}$ & PEAT, PEES & $\begin{array}{l}\text { Ocasionalmente } \\
\text { PEAT }\end{array}$ \\
\hline Alteración endocrina & Addison & Addison & $\begin{array}{c}\text { Addison e } \\
\text { hipogonadismo }\end{array}$ & Addison \\
\hline Exploración alterada & + & Frecuente & - & - \\
\hline Progresión & Rápida & $\begin{array}{c}\text { Rápida, } \\
\text { ocasionalmente } \\
\text { lenta }\end{array}$ & $\begin{array}{l}\text { Lenta, } \\
\text { ocasionalmente } \\
\text { rápida }\end{array}$ & - \\
\hline
\end{tabular}

ALD Adol: Adrenoleucodistrofia del adolescente; ALD Adul: Adrenoleucodistrofia del adulto; AMN: Adrenomieloneuropatía; PEV: Potenciales evocados visuales; PEAT: Potenciales evocados auditivos; PEES: Potenciales evocados somestésicos.

una gran heterogeneidad clínica. Los órganos que tienen mayor contenido en glucógeno son el hígado y el músculo esquelético, por lo que son estos órganos los más frecuente y gravemente afectados; a este respecto las glucogenosis se clasifican en hepáticas, musculares o generalizadas.

El hígado es el órgano encargado de mantener la homeostasis de la glucosa sanguínea. Cuando esta función está alterada, ello se manifiesta durante el ayuno con hipoglucemia y cetosis, pudiendo acompañarse o no de hepatomegalia. Si hay afectación muscular, donde el glucógeno es una fuente importante de energía para su contracción, los síntomas serán intolerancia al ejercicio, fatigabilidad, calambres y debilidad progresiva.

La edad de presentación en este grupo de enfermedades varía desde la infancia hasta la edad adulta ${ }^{2,19}$.

\section{Glucogenosis hepáticas}

Dentro de las glucogenosis que afectan predominantemente al hígado, la mayoría debutan en la época neonatal con la clínica antes referida. La glucogenosis tipo IV se caracteriza por tener una forma de presentación diferente en la edad adulta. Esta glucogenosis, debida al déficit de la enzima ramificante, cursa con el acumulo de agregados fibrilares típicos de la amilopectina que en la infancia generan un cuadro severo con afectación muscular y hepática, que evoluciona a la muerte en 4-5 años por fallo hepático o hemorragia gastrointestinal. En la edad adulta, se presenta con disfunción progresiva del SNC y periférico, alteración sensitiva, vejiga neurógena y demencia de inicio tardío. La edad de inicio se sitúa en la quinta o sexta década de la vida y el curso puede variar entre 3 y 20 años. En la biopsia del nervio periférico se aprecian los depósitos de poliglucosanos ${ }^{20,21}$.

\section{Glucogenosis musculares}

Los defectos en los enzimas responsables del sistema glucógeno fosforilasa forman un grupo heterogéneo de enfermedades clínica y genéticamente. El déficit de 
fosforilasa $b$ quinasa del músculo debuta en la adolescencia o edad adulta siendo muy infrecuente en la infancia. Se trata de una enfermedad con sintomatología restringida al músculo con calambres inducidos por el ejercicio y mioglobinuria o debilidad muscular progresiva de predominio distal y atrofia. Hay pacientes descritos sin intolerancia al ejercicio y casos en los que la clínica comienza en la infancia con debilidad muscular. La actividad de la fosforilasa b quinasa se encuentra disminuida en músculo pero en hígado y células sanguíneas es normal. La biopsia muscular muestra una miopatía vacuolar con numerosos depósitos de glucógeno ${ }^{20,22}$.

La forma más frecuente de presentación de los trastornos del glucógeno en la edad adulta es la glucogenosis tipo V (deficiencia de la fosforilasa muscular) o enfermedad de McArdle. Los síntomas suelen aparecer en la segunda y tercera década de la vida, aunque hay casos que debutan en la infancia y otros en pacientes que no presentan clínica hasta edades muy avanzadas. La clínica se caracteriza por intolerancia al ejercicio y calambres musculares, pudiendo desarrollar posteriormente fatiga y disminución de fuerza. Los desencadenantes son el ejercicio intenso y prolongado, siendo característico de esta enfermedad que se produzca el fenómeno en el que después de hacer un pequeño descanso, retoman el ejercicio con mayor facilidad. Aproximadamente la mitad de los pacientes, después de realizar un ejercicio intenso, presentan mioglobinuria por rabdomiolisis; en algunos casos ésta puede llegar a generar una situación de insuficiencia renal aguda.

La glucogenosis tipo VII, debida a la deficiencia de la fosfofructoquinasa muscular, también se puede diagnosticar en el adulto. Clínicamente es semejante a la enfermedad de McArdle, aunque suele ser más precoz y severa. Presentan intolerancia al ejercicio con dolor, calambres musculares y mioglobinuria con el ejercicio vigoroso, pero tiene ciertas características que la diferencian como la presencia de hiperuricemia o anemia hemolítica compensada. Hay una variante del adulto caracterizada por debilidad muscular len- tamente progresiva, sin calambres ni mioglobinuria ${ }^{20}$.

A veces, cuadros de miocardiopatía hipertrófica o aneurismas ventriculares pueden asociarse a glucogenosis generalizadas en las que se produce un acúmulo de glucógeno en el miocardio ${ }^{23}$.

Estas patologías se diagnostican mediante estudios enzimáticos en músculo, aunque en enfermedades como la de McArdle, cuya mutación genética es conocida, puede evitarse la biopsia.

\section{Mitocondriopatías}

Las mitocondriopatías se deben a mutaciones esporádicas o heredadas que afectan a genes localizados en el ADN nuclear o mitocondrial; la mayoría se dan por mutaciones del ADN nuclear. La frecuencia de este grupo de enfermedades es difícil de calcular aunque cada vez tenemos conocimento de más síndromes relacionados $^{24}$. En el adulto la prevalencia de mutaciones del ADN mitocondrial se estima en $1: 50.000^{25}$.

La edad de comienzo de las enfermedades mitocondriales puede situarse en un periodo que abarca desde la embriogénesis precoz a la edad adulta tardía.

Generalmente, las mitocondriopatías tienen un curso crónico, con progresión lenta, y se presentan como trastornos multisistémicos que se manifiestan principalmente en los tejidos y órganos; que tienen un consumo elevado de oxígeno como el músculo esquelético, el sistema nervioso central y periférico, el sistema endocrino, el miocardio, los ojos, los oídos, el aparato digestivo, los riñones o la médula ósea. Se caracterizan por una gran heterogenicidad clínica y no suele haber una sintomatología patognomónica diagnóstica aunque lo más habitual es que se presenten como enfermedades neuromusculares ${ }^{26}$. Debemos considerar esta posibilidad diagnóstica en todo paciente con una enfermedad crónica, intermitente o progresiva, con afectación simple o multisistémica no explicada por una enfermedad conocida ${ }^{25,27}$.

No hay un estándar diagnóstico para identificarlas lo que hace que, en muchos casos, la confirmación de que estemos 
ante una enfermedad mitocondrial suponga un importante reto para el clínico ${ }^{28,29}$. El procedimiento diagnóstico debe ser individualizado en cada caso y dado que se trata de enfermedades progresivas, una prueba de laboratorio normal en un momento dado de la evolución no permite descartarla, requiriéndose un seguimiento del paciente regular y exhaustivo. En la figura 3 se presenta una propuesta de algoritmo diagnóstico para las enfermedades mitocondriales.

Las mitocondriopatías que más frecuentemente se ven en los adultos son las derivadas de los defectos en la cadena respiratoria mitocondrial. Algunas de las características de los síndromes específicos de estas enfermedades con inicio en la edad adulta están descritas en la tabla 4 . Uno de los síndromes mitocondriales más

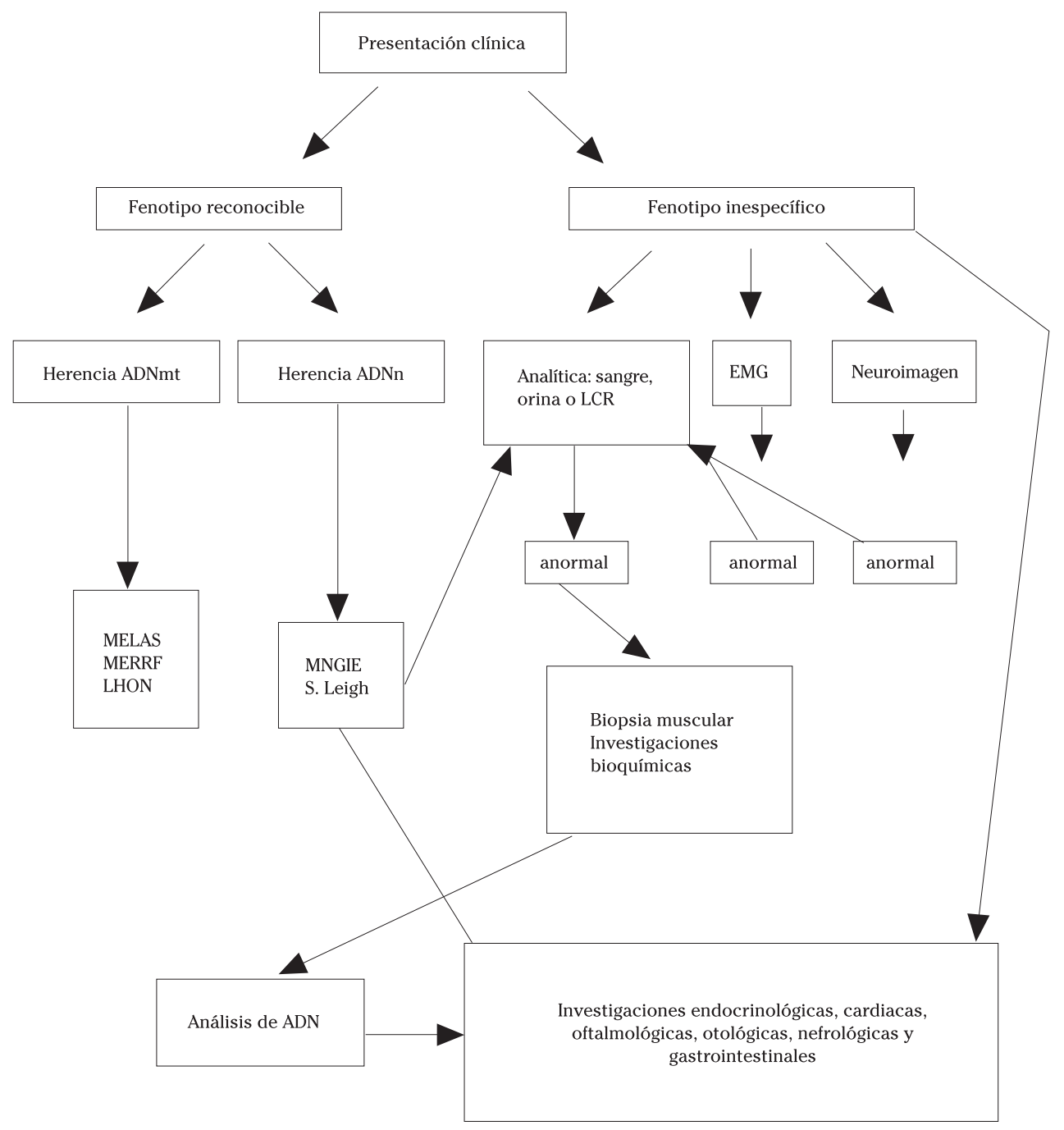

Figura 3. Algoritmo diagnóstico ante la sospecha de enfermedad mitocondrial. 
importantes por su frecuencia y por la repercusión que tienen los episodios ictales en este grupo de edad, es el MELAS, encefalomiopatía que cursa con episodios de déficit neurológico transitorio que simulan accidentes cerebrovasculares con hemiparesia y/o hemianopsia o ceguera cortical. Estos episodios aparecen invariablemente antes de los 40 años por lo que esta enfermedad debe entrar siempre en el diagnóstico diferencial del ictus en pacientes jóvenes, pudiendo ir acompañados de convulsiones, migraña, nauseas y vómitos.

Es frecuente encontrar una acidosis láctica durante el episodio o tras el ejercicio, siendo característico además que las lesiones anatómicas cerebrales, detectables durante los episodios no se correspondan con territorios vasculares de las grandes $\operatorname{arterias}^{24}$.

\section{OTROS ECM PREVALENTES EN LA EDAD ADULTA}

\section{Síndrome de Segawa o distonía dependiente de L-dopa}

La distonía dependiente de L-dopa o síndrome de Segawa se debe al déficit de GTP ciclohidrolasa ${ }^{30}$. Esta deficiencia limita la síntesis de tetrahidrobiopterina (BH4), que es el cofactor de TH, limitando así su actividad sobre el metabolismo de la dopamina en el sistema nigroestriado. Su herencia es autosómica dominante con penetrancia incompleta, siendo más frecuente en mujeres. El gen se localiza en el cromosoma 14, con gran heterogeneidad alélica y sin encontrarse historia familiar de distonía en la mitad de los pacientes, lo que sugiere un alto número de mutaciones espontáneas. La forma clásica aparece a los 5-6 años, como una distonía que afecta a extremidades inferiores con una fluctuación diurna característica de la clínica.

En la edad adulta, la forma de inicio se expresa como parkinsonismo. Generalmente, los pacientes han tenido alguna clínica leve desde la infancia. A diferencia de las formas infantiles hay una ausencia de progresión de la enfermedad. Algunos autores sugieren que las 2 formas, distonía y parkinsonismo, no son más que una diferente expresión clínica del mismo trastorno, pero en etapas evolutivas diferentes. También se describen en familiares de pacientes con esta patología un gran número de trastornos psiquiátricos como depresión, ansiedad, trastorno obsesivo-compulsivo, y trastornos de la alimentación ${ }^{31}$. La respuesta al tratamiento con L-dopa es excelente.

\section{Enfermedad de Wilson}

La enfermedad de Wilson o degeneración hepato-lenticular es un trastorno autosómico recesivo, caracterizado por cambios degenerativos en el cerebro, hepatopatía y anillos de Kayser-Fleischer en la córnea. El gen alterado se encuentra en el cromosoma 13. La patología se produce debido a una movilización defectuosa del cobre desde los lisosomas de los hepatocitos para su excreción a la bilis, por lo que se va acumulando en el hígado, hasta que la capacidad de retención de éste se ve superada, saliendo el cobre del interior del hígado para dañar otros órganos ${ }^{32}$. Han sido identificadas numerosas mutaciones en el gen, de tal manera que unas destruyen por completo la función del gen y se asocian con el comienzo de la clínica a los 2-3 años de edad, y otras mutaciones más ligeras pueden asociarse con síntomas neurológicos o hepatopatía a una edad tan tardía como a los 50 años $^{33}$. La forma de presentación más probable cuanto menor es la edad del paciente, es la de afectación hepática. En la adolescencia o la edad adulta aparecen las formas neurológicas o psiquiátricas, que cursan de manera brusca o insidiosa, con deterioro del rendimiento académico, alteraciones del comportamiento, temblor intencional, disartria o distonía $^{34,35}$. La sospecha clínica se confirma estudiando los marcadores del metabolismo del cobre. Los familiares de pacientes diagnosticados con la enfermedad deben ser estudiados para descartar la enfermedad de Wilson antes de la aparición de la sintomatología.

\section{BIBLIOGRAFIA}

1. Saudubray JM, Nuoffer JM, De Lonlay $P$, Castelnau P, Touati G. Hereditary metabolic diseases in adults. Rev Med Int 1008; 19 (Suppl 3): 366S-375S. 
2. Gray R, Preece M, Green S, Whitehouse W, WINER J, GREEN A. Inborn errors of metabolism as a cause of neurological disease in adults: an approach to investigation. J Neurol Neurosurg Psychiatry 2000; 69: 5-12.

3. Hoffman G, Nyhan W, Zschocke J, Kahler S, MAYATEPEK E. Inherited Metabolic Diseases. Ed: Lippincott Williams and Wilkins 2002: 250-252.

4. Sedel F, Barnerias C, Dubourg O, Desguerres I, LyON-CAEN O, SADUdRAY JM. Peripheral neuropathy and inborn errors of metabolism in adults. J Inherit Metab Dis 2007; 30: 642-653.

5. Sedel F, Gourfinkel An I, Lyon-Caen O, Baulac M, Saudubray JM, Navarro V. Epilepsy and inborn errors of metabolism in adults. a diagnostic approach. J Inherit Metab Dis 2007; 30: 846-854.

6. Cruysberg JRM, Borres GHJ, Trijbels JMC, Deutman AF et al. Delay in the diagnosis of homocystinuria : retrospective study of consecutive patients. BMJ 1996; 313: 10371040.

7. Hageman A, GabreËls F, De Jong J, GabreËlsFesten A, van den Berg C, van Oost B et al. Clinical symptoms of adult metacchromatic lukodystrophy and arylsulfatase A pseudodeficiency. Arch Neurol 1995; 52: 408-413.

8. Chabas A, Castellvi S, Akli S, Crosnier JM, Puech JP, Kahn A et al. Frequency of the arylsulphatase A pseudodeficiency allele in the Spanish population. Clin Genet 1993; 44 : 320-323.

9. Satoh JL, Tokumoto H, Kurohara K, Yukitake M, Matsui M, KuRoda Y et al. Adult-onset Krabbe disease with homozygous T1853C mutation in the galactocerebrosidase gne. Neurology 1997; 49: 1392-1399.

10. Felice KJ, Alessi AG, Grumnet ML. Clinical variability in adult-onset acid maltase deficiency: report of affected sibs and review of the literature. Medicine 1995; 74: 131-135.

11. Gould SJ, Raymond GV, Valle D. The Peroxisome Biogenesis Disorders. En: Scriver CR, Beaudet AL, Sly WS, Valle D (editors). The Metabolic \& Molecular Bases of Inherited Disease.New York: McGraw-Hill; 2001: 3181-3218.

12. Raymond G.V. Peroxisomal disorders. Curr Opin Neurol 2001; 14: 783-787.

13. WANDERS RJA, JAKOBS C, SKJELdAL OH. Refsum Disease. En: Scriver CR, Beaudet AL, Sly WS, valle D (editors). The Metabolic \&
Molecular Bases of Inherited Disease. New York: McGraw-Hill, 2001: 3303.

14. Van Geel BM, Bezman L, Loes DJ, Moser HW, RAYMOND GV. Evolution of phenotypes in aduld male patients with X-linked adrenoleukodystrophy. Ann Neurol 2001; 49: 186-194.

15. MOSER HW. Adrenoleukodystrophy: phenotype, genetics, pathogenesis and therapy. Brain1997; 120: 1485-1508.

16. Moser HW, Smith KD, Watkins PA, Powers J, Moser AB. X-Linked Adrenoleucodystrophy. En: Scriver CR, Beaudet AL, Sly WS, Valle D (editors). The Metabolic \& Molecular Bases of Inherited Disease. New York: McGrawHill, 2001: 3257-3302.

17. Van Geel BM, Assies J, Wanders RJ, Barth PG. $\mathrm{X}$ linked adrenoleukodystrophy: clinical presentation, diagnosis, and therapy. J. Neurol. Neurosurg. Psychiatry1997; 63: 4-14

18. Moser HW, RAYMond GV. Genetic Peroxisomal Disorders: Wy, When, and How to Test. Ann Neurol 1998; 44: 713-714.

19. Chen YT. Glycogen Storage Diseases. En: Scriver CR, Beaudet AL, Sly WS, Valle D (editors). The Metabolic \& Molecular Bases of Inherited Disease. New York: McGrawHill, 2001: 1521.

20. Bruno C, Servidei S, Shanske S, Karpati G, CARPenter S, McKeE D et al. Glycogen branching enzyme deficency in adult polyglucosan body disease. Ann Neurol 1993; 33: 88-93.

21. Clemens PR, Yamamoto M, Engel AG. Adult phosphorylase b kinase deficiency. Ann Neurol 1990; 28: 529-538.

22. Arad M, Maron BJ, Gorham JM, Johnson WH, SAUl JP, PEREz-ATAYde AR et al. Glycogen storage diseases presenting as hipertrophic cardiomyopathy. N Engl J Med 2005; 352: 362-372.

23. Toda G, Yoshimuta T, Kawano H, Yano $\mathrm{K}$. Glycogen storage disease associated with left ventricular aneurysm in an elderly patient. Jpn Circ J 2001; 65: 462-464.

24. SmeitinK JAM. Mitochondrial disorders: Clínical presentation and diagnostic dilemas. J Inherit Metab Dis 2003; 26: 199207.

25. FinStERER J. Mitocondriopatías (MCP). Eur J Neurol (Ed. Esp.) 2004; 3: 110-133.

26. Munnich A, Rötig A, Chretien D, Sadudray J, CORMIER V, Rustin P. Clinical presentations and laboratory investigations in respiratory chain deficiency. Eur J Pediatr 1996; 155: 262-274. 
27. Bernier FP, Boneh A, Dennett X, Chow CW, Cleary MA, Thorburn DR. Diagnostic criteria for respiratory chain disorders in adults and children. Neurology 2002; 59: 14061411.

28. Pien K, van Vlem B, van Coster R, Dacremont G, PIETTE M. An Inherited metabolic disorder presenting as ethylene glycol intoxication in a young adult. Am J Forensic Med Pathol 2002; 23: 96-100.

29. Finisterer J, Jarius C, Eichberger $\mathrm{H}$. Phenotype variability in 130 adult patients with respiratory chain disorders. J Inherit Metab Dis 2001; 24: 560-576.

30. Segawa M, Nomura Y, NishiYama N. Autosomal dominat guanosine triphosphate cyclohydrolase I deficiency (Segawa disease). Ann Neurol 2003; 54 (Suppl. 6): S32-S45.

31. Hanh H, Trant MR, Brownstein MJ, Harper RA, Milstien S, Butler IJ. Neurologic and psychiatric manifestations in a family with a mutation in exon 2 of the guanosine triphosphate-cyclohydrolase gene. Arch Neurol 2001; 58: 749-755.

32. Bull PC, Thomas GR, Rommens JM, Forbes JR, Cox DW. The Wilson disease gene is a putative copper transporting P-type ATPase similar to the Menkes gene. Nat Genet 1993; 5: 327-337.

33. Thomas GR, Roberts EA, Walshe JM, Cox DW. Haplotypes and mutations in Wilson disease. Am J Hum Genet 1995; 56: 13151319.

34. Gollan JL, Gollan TJ. Wilson's disease in 1998: genetic, diagnostic and therapeutic aspects. J Hepatol 1998; 28 (Suppl. 1): 28-36.

35. Gow PJ, Smallwood RA, Angus PW, Smith AL, Wall AJ, SEWEll RB. Diagnosis of Wilson's disease: an experience over three decades. Gut 2000; 46: 415-418. 
